International Journal of Pure and Applied Mathematics

Volume 95 No. 3 2014, 427-452

ISSN: 1311-8080 (printed version); ISSN: 1314-3395 (on-line version)

url: http://www.ijpam.eu

doi: http://dx.doi.org/10.12732/ijpam.v95i3.10

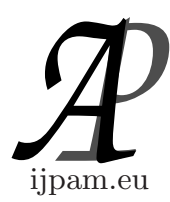

\title{
THE SINGLE-VALUED EXTENSION PROPERTY AND \\ BISHOP'S PROPERTY ( $\beta)$ FOR CERTAIN \\ GENERALIZED CLASSES OF OPERATORS \\ ON HILBERT SPACES
}

\author{
Ould Ahmed Mahmoud Sid Ahmed \\ Department of Mathematics \\ College of Science \\ Al Jouf University \\ Al Jouf, 2014, KINGDOM OF SAUDI ARABIA
}

\begin{abstract}
An operator $T \in \mathcal{L}(\mathcal{H})$ is called of class $[n Q N]$ if $T^{n}\left(T^{*} T\right)=$ $\left(T^{*} T\right) T^{n}$ for a positive integer $n$, which is a common generalization of the quasi-normal and normal operators classes. Several properties of such class have been studied by the author in [22] and [23]. In this paper it is proved that in order to find a nontrivial subspace for a $n$-power quasi-normal operator $T$ it suffices to make the further assumption that $\sigma(T) \bigcap \sigma\left(\omega_{k}^{-1} T\right)=\emptyset$ where $\omega_{k}=\exp \left(i \frac{2 k \pi}{n}\right)$ for $k=1,2, \ldots, n-1$, (i.e., $\left.\omega_{k}^{n}=1\right)$.
\end{abstract}

AMS Subject Classification: 47B20, 47B15

Key Words: $n$-power quasi-normal, $m$-partial isometry-subscalar, single valued extension property (SVEP), Bishop's property, approximate spectrum, spectrum

\section{Introduction}

Let $\mathcal{H}$ be an infinite dimensional separable complex Hilbert space, $\mathcal{K}$ be a complex Hilbert space and denote by $\mathcal{L}(\mathcal{H}, \mathcal{K})$ the algebra of all bounded linear

Received: May 3, 2014

(c) 2014 Academic Publications, Ltd. url: www.acadpubl.eu 
operators from $\mathcal{H}$ to $\mathcal{K}$. We write $\mathcal{L}(\mathcal{H})$ for $\mathcal{L}(\mathcal{H}, \mathcal{H})$. If $T \in \mathcal{L}(\mathcal{H}, \mathcal{K})$ we shall write $\mathcal{N}(T)$ and $\mathcal{R}(T)$ for the null space and the range of $T$, respectively. We shall denote the set of all complex numbers and the complex conjugate of a complex number $\lambda$ by $\mathbb{C}$ and $\bar{\lambda}$, respectively. The closure of a set $\mathcal{M}$ will be denoted by $\overline{\mathcal{M}}$, and we shall henceforth shorten $\lambda I-T$ by $\lambda-T$. The spectrum, the point spectrum, the approximate point spectrum of an operator $T$ are denoted by $\sigma(T), \sigma_{p}(T), \sigma_{a p}(T)$ respectively. $T^{*}$ means the adjoint of $T$. An operator $T$ is said to be positive (denoted by $T \geq 0$ ) if $\langle T x \mid x\rangle \geq 0$ for all $x \in \mathcal{H}$.

The study of operators satisfying Bishop's property $(\beta)$ is of significant interest and is currently being done by a number of mathematicians around the world.Recently many mathematicians are working on finding operators which have Bishops property, SVEP and which satisfy Weyl's theorem. One of the most important subclasses of the algebra of all bounded linear operators acting on Hilbert space, the class of hyponormal operators $\left(T^{*} T \geq T T^{*} \Longleftrightarrow|T|^{2} \geq\right.$ $\left|T^{*}\right|^{2}$ ), has been studied by many authors (see [31]). In recent years this class has been generalized, in some sense, to the larger sets of so-called $k$-quasiclass $\mathcal{A}, p$-hyponormal, $p$-quasihyponormal, $k$-quasinormal operators and $(p, k)$ quasihyponormal.

Recall that an operator $T \in \mathcal{L}(\mathcal{H})$ is called:

(1) A class $\mathcal{A}$ operator iff $\left|T^{2}\right| \geq|T|^{2}$ where $|T|=\left(T^{*} T\right)^{\frac{1}{2}}$ (see [9]).

(2) A quasi-class $\mathcal{A}$ operator iff $T^{*}\left|T^{2}\right| T \geq T^{*}|T|^{2} T$ (see [11]).

(3) $k$-quasi- class $\mathcal{A}$ operator iff $T^{* k}\left(\left|T^{2}\right|-|T|^{2}\right) T^{k} \geq 0$ (see [26]).

(4) $k$-quasi-*- class $\mathcal{A}$ operator iff $T^{* k}\left(\left|T^{2}\right|-\left|T^{*}\right|^{2}\right) T^{k} \geq 0 \quad$ (see [20]).

(5) $p$-hyponormal iff $\left(T^{*} T\right)^{p}-\left(T T^{*}\right)^{p} \geq 0,0<p \leq 1 \quad$ (see [2]).

(6) $p$-quasihyponormal iff $T^{*}\left(\left(T^{*} T\right)^{p}-\left(T T^{*}\right)^{p}\right) T \geq 0, \quad 0<p<1 \quad$ (see [3])

(7) $k$-quasihyponormal iff $T^{* k}\left(T^{*} T-T T^{*}\right) T^{k} \geq 0, k$ is a positive integer (see $[6])$.

(8) $\quad(p, k)$-quasihyponormal iff $T^{* k}\left(\left(T^{*} T\right)^{p}-\left(T T^{*}\right)^{p}\right) T^{k} \geq 0,0<p<$ $1, k$ is a positive integer (see [17]). 
(9) $m$-isometry iff

$$
T^{* m} T^{m}-\left(\begin{array}{c}
m \\
1
\end{array}\right) T^{* m-1} T^{m-1}+\left(\begin{array}{c}
m \\
2
\end{array}\right) T^{* m-2} T^{m-2}-\ldots+(-1)^{m} I=0 \text { (see[1]). }
$$

A $k$-quasi-class $\mathcal{A}$ operator is an extension of class $\mathcal{A}(k=0)$ and quasiclass $\mathcal{A}(k=1)$. It was known that these operators share many interesting properties with hyponormal operators (see $[10,11,12]$ ).

A $(p, k)$-quasihyponormal operator is an extension of $p$-hyponormal, $k$ quasihyponormal and $p$-quasihyponormal. A 1-hyponormal operator is called a hyponormal operator, which has been studied by many authors and it is known that hyponormal operators have many interesting properties similar to those of normal operators (see [31]).

In 1984, M.Putinar showed in [25] that every hyponormal operators and more generally subscalar operators possess Bishop's property $(\beta)$, and then 1987 ,S.Brown used this results to prove that a hyponormal operator with rich spectrum has a non trivial subspace ( see [5]). These results were generalized to $k$-quasi-class $\mathcal{A}$ operators (see $[12,21,26,30])$ and to $(p, k)$-quasihyponormal operatoers (see $[8,13,17,30])$

The purpose of this paper is to make a beginning on the invariant subspace problem for another class of operators closely related to the quasi-normal and normal operators namely, the class of $n$-power quasi-normal.

An operator $T \in \mathcal{L}(\mathcal{H})$ is said to be $n$-power quasi-normal (abbreviated as $n Q N), n=1,2, \ldots$, if $T^{n} T^{*} T=T^{*} T T^{n}$. If $n=1, T$ is called quasi-normal. This class of operators being denoted by $[n Q N]$, i.e.

$$
[n Q N]:=\left\{T \in \mathcal{L}(\mathcal{H}) / T^{n} T^{*} T-T^{*} T T^{n}=0\right\},
$$

was studied by author $[22,23]$.

$T$ is called an $m$-partial isometry if $T$ satisfies

$$
T B_{m}(T)=T \sum_{k=0}^{m}\left(\begin{array}{c}
m \\
k
\end{array}\right)(-1)^{k} T^{* m-k} T^{m-k}=0,
$$

where $B_{m}(T)$ is obtained formally from the binomial expansion of $B_{m}(T)=$ $\left(T^{*} T-I\right)^{m}$ by understanding $\left(T^{*} T\right)^{m-k}=T^{* m-k} T^{m-k}$. The case when $m=1$ is called the partial isometries class. The class of $m$-partial isometries was defined by A.Saddi and O.A.M. Sid Ahmed [27] who proved some properties of the class. See Proposition 6.1. 
The following characterization of 3-isometry is given by M. Scott in [28]. An operator $T \in \mathcal{L}(\mathcal{H})$ is a 3 -isometry if

$$
T^{* 3} T^{3}-3 T^{* 2} T^{2}+3 T^{*} T-I=0 .
$$

Equivalently $T$ is a 3 -isometry if and only if there exist operators $B_{1}\left(T^{*}, T\right)$ and $B_{2}\left(T^{*}, T\right)$ such that for all natural numbers $n$,

$$
T^{* n} T^{n}=I+n B_{1}\left(T^{*}, T\right)+n^{2} B_{2}\left(T^{*}, T\right) .
$$

In this case it is straightforward to verify that

$$
2 B_{2}\left(T^{*}, T\right)=T^{* 2} T^{2}-2 T^{*} T+I
$$

and

$$
2 B_{1}\left(T^{*}, T\right)=-T^{* 2} T^{2}+4 T^{*} T-3 I .
$$

Evidently, each $B_{j}\left(T^{*}, T\right)$ is self-adjoint.

This article is been divided into six sections. Section 2 deals with some preliminary facts concerning functional spaces. In Section 3 we continue the study of operators in $[n Q N]$ and introduce the the class of $n$-power $k$-quasinormal operators. Section 4 includes our main results. We show that some of class $[n Q N]$ satisfy an analogue of the single valued extension for $W_{2}^{m}(\mathcal{D} ; \mathcal{H})$ and have scalar extension. In Section 5, we give an analogous result for the Berberian extension. Finally we prove that if $T$ is invertible operator such that $T$ and $T^{*}$ are $m$-partial isometry for $m \geq 2$, then $T$ has Bishop's property $(\beta)$ if and only if $T^{*}$ has Bisop's $(\beta)$.

\section{Vector Valued Functions Spaces}

We will need the following functional spaces:

Let $\lambda$ be the coordinate in $\mathbb{C}$ and let $d \mu(\lambda)$ denotes planar Lebesgue measure. Let $\mathcal{D}$ be a bounded open subset of $\mathbb{C}$. We shall denote by $L^{2}(\mathcal{D} ; \mathcal{H})$ the Hilbert space of measurable function $f: \mathcal{D} \longrightarrow \mathcal{H}$ such that

$$
\|f\|_{2, \mathcal{D}}=\left\{\int_{\mathcal{D}}\|f(\lambda)\|^{2} d \mu(\lambda)\right\}^{\frac{1}{2}}<\infty .
$$

The space of functions $f \in L^{2}(\mathcal{D}, \mathcal{H}$ ) that are analytic in $\mathcal{D}$ (i.e., $\bar{\partial} f=$ $\left.\frac{\partial f}{\partial \bar{z}}=0\right)$ is denoted by

$$
A^{2}(\mathcal{D} \mathcal{H})=L^{2}(\mathcal{D}, \mathcal{H}) \cap O(\mathcal{D}, \mathcal{H})
$$


where $O(\mathcal{D}, \mathcal{H})$ denote the space of $\mathcal{H}$-valued analytic functions on $\mathcal{D}$ equipped with the topology of uniform convergence on compact subsets of $\mathcal{D}$. $O(\mathcal{D}, \mathcal{H})$ is a Frechet space.

The set $A^{2}(\mathcal{D}, \mathcal{H})$ is called the Bergman space for $\mathcal{D}$. Note that $A^{2}(\mathcal{D}, \mathcal{H})$ is a Hilbert space. We denote by $P$ the orthogonal projection of $L^{2}(\mathcal{D}, \mathcal{H})$ onto $A^{2}(\mathcal{D}, \mathcal{H})$.

Let us define now a special Sobolev type space. Let $m$ be a fixed nonnegative integer. The vector valued Sobolev space $W_{2}^{m}(\mathcal{D}, \mathcal{H})$ with respect to $\bar{\partial}$ and of order $m$ will be the space of those functions $f \in L^{2}(\mathcal{D}, \mathcal{H})$ whose derivatives $\bar{\partial} f, \ldots, \bar{\partial}^{m} f$ in the sense of distributions also belong to $L^{2}(\mathcal{D}, \mathcal{H})$, i.e,

$$
W_{2}^{m}(\mathcal{D}, \mathcal{H})=\left\{f \in L^{2}(\mathcal{D}, \mathcal{H}): \bar{\partial}^{k} f \in L^{2}(\mathcal{D}, \mathcal{H}), \text { for }, \quad k=0,1, \ldots, m\right\}
$$

Endowed with the norm

$$
\|f\|_{W_{2}^{m}}^{2}=\sum_{k=0}^{m}\left\|\bar{\partial}^{k} f\right\|_{2, \mathcal{D}}^{2}
$$

$W_{2}^{m}(\mathcal{D}, \mathcal{H})$ becomes a Hilbert space contained continuously in $L^{2}(\mathcal{D}, \mathcal{H})$.

Definition 2.1. We say that $T$ has the single valued extension property at $\lambda_{0} \in \mathbb{C}$ (abbreviated SVEP at $\lambda_{0}$ ) if for every open neighborhood $U$ of $\lambda_{0}$, the only analytic solution $\mathrm{f}$ to the equation $(T-\lambda) f(\lambda)=0$ for all $\lambda$ in $U$ is the constant function $f \equiv 0$.

We say that $T$ has SVEP if $T$ has a SVEP at every $\lambda \in \mathbb{C}$.

The SVEP of $T \in \mathcal{L}(\mathcal{H})$ may by also defined as follows : Every $T \in \mathcal{L}(\mathcal{H})$ induces a continuous mapping $T_{\mathcal{D}}$ on $O(\mathcal{D}, \mathcal{H})$ defined by

$$
T_{\mathcal{D}}(f)(\lambda)=(\lambda-T) f(\lambda) \text { for all } f \in O(\mathcal{D}, \mathcal{H}) .
$$

The operator $T$ has SVEP precisely when $T_{\mathcal{D}}$ is one-to-one.

The SVEP may be characterized by means of some typical tools originating from the local spectral theory. Recall that, for a bounded operator $T$ on $\mathcal{H}$, the local resolvent set $\rho_{T}(x)$ of $T$ at the point $x \in \mathcal{H}$,is defined as the union of all open subsets $\mathcal{D}$ of $\mathbb{C}$ such that there exists an analytic function $f: D \longrightarrow \mathcal{H}$ which satisfies

$$
(\lambda-T) f(\lambda)=x \text { for all } \lambda \in \mathcal{D} .
$$

The local spectrum $\sigma_{T}(x)$ of $T$ at $x \in \mathcal{H}$ is the set defined by $\sigma_{T}(x):=\mathbb{C} \backslash \rho_{T}(x)$ and obviously $\sigma_{T}(x) \subset \sigma(T)$. It is clear from the definition that, $T$ has SVEP 
if and only if zero is the unique vector $x \in \mathcal{H}$ such that $\sigma_{T}(x)=\emptyset$ (see for more details [19]).

Recall, [24, Corollary 2.24], that a necessary and sufficient condition for a surjective operator $T \in \mathcal{L}(\mathcal{H})$ to be invertible is that $T$ has SVEP at 0 .

Definition 2.2. An bounded operator $T \in \mathcal{L}(\mathcal{H})$ is said to have the Bishop's property $(\beta)$ if, for every open subset $\mathcal{D}$ of the complex plane $\mathbb{C}$ and every sequence of analytic functions $f_{n}: U \longrightarrow \mathcal{H}$ with the property that

$$
(\lambda-T) f_{n}(\lambda) \longrightarrow 0 \text { as } n \longrightarrow \infty
$$

uniformly on all compact subset of $\mathcal{D}, f_{n}(\lambda) \longrightarrow 0$ as $n \longrightarrow \infty$ locally uniformly on $\mathcal{D}$, or equivalent to for every open subset $\mathcal{D}$ of $\mathbb{C}$ the operator $T_{\mathcal{D}}$ defined in (2.1) is one-to-one and has close range.

It is a very important property in spectral theory. It is well-known that every normal operator $\left(T^{*} T=T T^{*}\right)$ has Bishop's property $(\beta)$.

The local spectral subspace for $T$ is defined by

$$
\mathcal{H}_{T}(F)=\left\{x \in \mathcal{H}: \sigma_{T}(x) \subset F\right\} \text { for } F \subset \mathbb{C} .
$$

$T \in \mathcal{L}(\mathcal{H})$ is said to have Dunford's property $(C)$ if $\mathcal{H}_{T}(F)$ is closed for each closed subset $F$ of $\mathbb{C}$. It is well known from [24] that

$$
\text { Property }(\beta) \Longrightarrow \text { Dunford's property }(C) \Longrightarrow \text { SVEP. }
$$

Definition 2.3. A bounded operator $T$ on $\mathcal{H}$ is called scalar of order $m$ if it possesses a spectral distribution of order $m$, i.e., if there is a continuous unital morphism,

$$
\Phi: C_{0}^{m}(\mathbb{C}) \longrightarrow \mathcal{L}(\mathcal{H}),
$$

such that $\Phi(z)=T$, where $z$ stands for the identity function on $\mathbb{C}$ and $C_{0}^{m}(\mathbb{C})$ for the space of compactly supported functions on $\mathbb{C}$, continuously differentiable of order $m, 0 \leq m \leq \infty$. An operator is subscalar if it is similar to the restriction of a scalar operator to an invariant subspace.

For $f \in C_{0}^{m}(\mathbb{C})$ let $M_{f}$ be the operator on $W_{2}^{m}(\mathcal{D} ; \mathcal{H})$ given by multiplication by $f$. This has a spectral distribution of order $m$, defined by the functional calculus

$$
\Phi_{M}: C_{0}^{m}(\mathbb{C}) \longrightarrow \mathcal{L}\left(W_{2}^{m}(\mathcal{D} ; \mathcal{H})\right) ; \Phi_{M}(f)=M f .
$$

Therefore $M_{f}$ is a scalar operator of order $m$. Consider a bounded open disk $\mathcal{D}$ which contains $\sigma(T)$ and the quotient space

$$
H(\mathcal{D})=\frac{W_{2}^{m}(\mathcal{D} ; \mathcal{H})}{\overline{(T-z) W_{2}^{m}(\mathcal{D} ; \mathcal{H})}}
$$


endowed with the Hilbert space norm. We denote the class of a vector $f$ or an operator A on $H(\mathcal{D})$ by $\widehat{f}$, respectively $\widehat{A}$. Let $M_{z}$ be the operator of multiplication by $z$ on $W_{2}^{m}(\mathcal{D} ; \mathcal{H})$. As noted above, $M_{z}$ is a scalar of order $m$ and has a spectral distribution $\Phi$. Let $S \equiv \widehat{M}_{z}$. Since $\overline{(T-z) W_{2}^{m}(\mathcal{D} ; \mathcal{H})}$ is invariant under every operator $M_{f} ; f \in C_{0}^{m}(\mathbb{C})$, we infer that $S$ is a scalar operator of order $m$ with spectral distribution $\widehat{\Phi}$. Consider the natural map $V: \mathcal{H} \longrightarrow \mathcal{H}(\mathcal{D})$ defined by $V h=[\widehat{1 \otimes h}]$, for $h \in \mathcal{H}$, where $1 \otimes h$ denotes the constant function identically equal to h. In [25], Putinar showed that if $T \in \mathcal{L}(\mathcal{H})$ is a hyponormal operator then $V$ is one-to-one and has closed range such that $V T=S V$, and so $T$ is subscalar of order $m$.

\section{The Class of $n$-Power $k$-Quasi-Normal Operators: $[(n, k) Q N]$ in $L(H)$}

\section{1. n-Power Quasi-Normal Operators}

In this section, we continue the study of operators in $[n Q N]$. It was shown that if $T$ is of class $[2 Q N] \cap[3 Q N]$ then $T$ is of class $[n Q N], n=4,5, \ldots$,and several structure theorems for operators in $[n Q N]$ were proved. Further, it was studied some cases in which an operator $T \in[n Q N]$ is quasi-normal, normal operators, $T^{n}$ is normal, and isometry.

Theorem 3.1. ([22]) Let $T \in \mathcal{L}(\mathcal{H})$

1. if $T \in[2 Q N] \cap[3 Q N]$ such that $\mathcal{N}(T) \subset \mathcal{N}\left(T^{*}\right)$, then $T$ is normal.

2. if $T \in[2 Q N] \cap[3 Q N]$ such that $T-I \in[n Q N]$, then $T$ is normal.

3. if $T$ and $T-I$ are of class class $[2 Q N]$, then $T$ is normal.

4. if $T \in[2 Q N]$ such that $0 \notin W(T)$ then $T$ is normal.

5. if $T \in[n Q N]$ such that $T$ is invertible, then $T^{n}$ is normal.

6. if both $T$ and $T^{*}$ are of class $[n Q N]$, then $T^{n}$ is normal.

7. if $T \in[n Q N]$ such that $\mathcal{N}\left(T^{*}\right) \subset \mathcal{N}(T)$ then $T^{n}$ is normal.

Theorem 3.2. ([22]) Let $T \in \mathcal{L}(\mathcal{H})$

1. if $T \in[2 Q N] \cap[3 Q N]$ such that $\mathcal{N}(T) \subset \mathcal{N}\left(T^{*}\right)$, then $T$ is quasi-normal. 
2. if $T \in[2 Q N]$ such that $T^{2} \in[2 Q N]$ et $\mathcal{N}(T) \subset \mathcal{N}\left(T^{*}\right)$, then $T^{2}$ is quasinormal.

3. if $T \in[2 Q N] \cap[3 Q N]$ such that $T^{2} \in[2 Q N]$, then $T^{2}$ is quasi-normal.

Proposition 3.1. ([23]) Let $T \in \mathcal{L}(\mathcal{H})$. then we have

1. if $T \in[n Q N] \cap[(n+1) Q N]$ such that $T$ or $T^{*}$ is injective, then $T$ is quasi-normal.

2. if $T \in[2 Q N]$ such that $T$ is a 2-isometry, then $T^{2} \in[n Q N], \quad n=$ $3,4,5, \ldots$.

Theorem 3.3. Let $T \in \mathcal{L}(\mathcal{H})$ such that $T$ is an 2-isometry. If $T^{2}$ is of class $[n Q N]$, then $T$ is of class $[2 n Q N]$.

Proof. Since $T^{2 n}\left(T^{* 2} T^{2}\right)=\left(T^{* 2} T^{2}\right) T^{2 n}$ and $T^{* 2} T^{2}=2 T^{*} T-I$ we have

$$
T^{2 n}\left(2 T^{*} T-I\right)=\left(2 T^{*} T-I\right) T^{2 n} \Longrightarrow T^{2 n} T^{*} T=T^{*} T T^{2 n} .
$$

Thus, $T$ is of class $[2 n Q N]$.

Theorem 3.4. Let $T$ is of class $[n Q N]$ such that $T$ is a 3-isometry. If

$$
T^{n}\left(T^{* 2} T^{2}\right)=\left(T^{* 2} T^{2}\right) T^{n},
$$

then $T^{k}$ is of class $[n Q N]$ for $k=1,2, \ldots$

Proof. Since $T$ is of class $[n Q N]$ we have by using (1.1)

$$
\begin{aligned}
\left(T^{k}\right)^{n}\left(T^{* k} T^{k}\right) & =\left(T^{k}\right)^{n}\left(I+k B_{1}\left(T^{*}, T\right)+k^{2} B_{2}\left(T^{*}, T\right)\right) \\
& =T^{n k}+k T^{n k} B_{1}\left(T^{*}, T\right)+k^{2} T^{n k} B_{2}\left(T^{*}, T\right) \\
& =T^{n k}+k B_{1}\left(T^{*}, T\right) T^{n k}+k^{2} B_{2}\left(T^{*}, T\right) T^{n k} \\
& =\left(I+n B_{1}\left(T^{*}, T\right)+n^{2} B_{2}\left(T^{*}, T\right)\right) T^{n k} \\
& =T^{* k} T^{k} T^{n k} .
\end{aligned}
$$

\section{2. $n$-Power $k$-Quasinormal Operators}

Definition 3.1. An operator $T \in \mathcal{B}(\mathcal{H})$ is called an $n$-power $q$-quasinormal operator or equivalently $(n, k)$-quasi-normal if

$$
T^{n}\left(T^{*} T\right)^{k}=\left(T^{*} T\right)^{k} T^{n}
$$

for some positive integers $n$ and $k$. This class of operators will denoted by $[(n, k) Q N]$. 
Remark 3.1. 1. $[(1,1) Q N]$ is the class of quasi-normal operator i.e. $[(1,1) Q N]=[Q N]$.

2. $[(n, 1) Q N]$ is the class of $n$-power quasi normal operators i.e., $[(n, 1) Q N]=$ $[n Q N]$.

3. $(1, k)$-quasi-normal is an $k$-quasi-normal. (see [29]).

4. Every $n$-power quasi-normal operator is an $(n, k)$-quasi-normal.

5. Every $k$-quasi-normal operator is an $(n, k)$-quasi-normal.

Example 3.1. Let $\left(e_{j}\right)_{j>0}$ be an orthogonal basis of a Hilbert space $\mathcal{H}$ and let $T \in \mathcal{B}(\mathcal{H})$ be a weight shift with non zero weight $\left(\omega_{j}\right)_{j>0}$. Then $T$ is an $(n, k)$-quasinormal if and only if

$$
\left|\omega_{j}\right|^{2 k}=\left|\omega_{j+n}\right|^{2 k}, j=0,1,2, \ldots
$$

In fact, since $T e_{j}=\omega_{j} e_{j+1}$ and $T^{*} e_{j}=\omega_{j} e_{j-1}$, a simple a computation shows that

$$
T^{n}\left(T^{*} T\right)^{k}\left(e_{j}\right)=\left|\omega_{j}\right|^{2 k} \omega_{j} \omega_{j+1} \ldots \omega_{j+n-1} e_{j+n}
$$

and

$$
\left(T^{*} T\right)^{k} T^{n}\left(e_{j}\right)=\omega_{j} \omega_{j+1} \ldots \omega_{j+n-1}\left|\omega_{j+n}\right|^{2 k} e_{j+n} .
$$

Hence

$$
T^{n}\left(T^{*} T\right)^{k}=\left(T^{*} T\right)^{k} T^{n} \Longleftrightarrow\left|\omega_{j}\right|^{2 k}=\left|\omega_{j+n}\right|^{2 k}, j=0,1,2, \ldots
$$

Remark 3.2. If $T$ is an (n.k)-quasi-normal, then $T$ is

- $(2 n, k)$-quasi-normal operator.

- $(n, 2 k)$-quasi-normal operator.

- $(2 n, 2 k)$-quasi-normal operator.

The following proposition gives a characterization of an $(n, k)$-power quasinormal operator.

Proposition 3.2. Let $T \in \mathcal{L}(H), A=T^{n}+\left(T^{*} T\right)^{k}$ and $B=T^{n}-\left(T^{*} T\right)^{k}$. Then $T$ is of class $[(n, k) Q N]$ if and only if $A$ commutes with $B$.

Proof. Commutativity of $A$ and $B$ is equivalent to $T^{n}\left(T^{*} T\right)^{k}=\left(T^{*} T\right)^{k} T^{n}$.

Proposition 3.3. Let $T, A, B$ be as in Proposition 3.2. Then if $T$ is of class $[(n, k) Q N]$, then $T^{n}\left(T^{*} T\right)^{k}$ commutes with $A$ and $B$. 
Proof. By (3.1) we have that

$$
T^{n} T^{*} T\left(T^{n} \pm T^{*} T\right)=\left(T^{n} \pm T^{*} T\right) T^{n} T^{*} T .
$$

In general, the two classes $[(n, k) Q N]$ and $[(n+1, k) Q N]$ are not the same (see [22]).

Proposition 3.4. If $T$ is both of class $[(n, k) Q N]$ and $[(n+1, k) Q N]$, then it is of class $[(n+2, k) Q N]$. i.e., $[(n, k) Q N] \cap[(n+1, k) Q N] \subset[(n+2, k) Q N]$.

Proof. By (3.1) we have that

$$
T^{n+1}\left(T^{*} T\right)^{k}=T^{n}\left(T^{*} T\right)^{k} T,
$$

so that $T^{n+2}\left(T^{*} T\right)^{k}$ may be transformed into $\left(T^{*} T\right)^{k} T^{n+2}$.

Proposition 3.5. If $T$ is of class $[(n, k) Q N]$ such that $T$ is a partial isometry, then $T$ is of class $[(n+1, k) Q N]$.

Proof. Since $T$ is a partial isometry, therefore

$$
T T^{*} T=T \text {. }
$$

and hence $\left(T^{*} T\right)^{k}=T^{*} T$. Multiplying (3.3) to the left by $T^{n}$ and from right by $\left(T^{*} T\right)^{k}$ and using the fact that $T$ is of class [ $\left.(n, k) N Q\right]$, we get

$$
\begin{aligned}
T^{n+1}\left(T^{*} T\right)^{k} & =T^{n+1}\left(T^{*} T\right)^{k-1} \\
& =T^{n+1}\left(T^{*} T\right)^{k} \\
& =T\left(T^{*} T\right)^{k} T^{n} \\
& =T\left(T^{*} T\right)^{k-1} T^{n} \\
& =\cdots \\
& =T^{n+1} \\
& =\left(T^{*} T\right)^{k} T^{n+1} .
\end{aligned}
$$

which implies that $T$ is of class $[(n+1, k) Q N]$.

The class $[(n, k) Q N]$ has the following properties

Theorem 3.5. The class $[(n, k) Q N]$ is closed under unitary equivalence and scalar multiplication.

Proof. Let $S \in \mathcal{L}(\mathcal{H})$ be unitary equivalent to $T$. Then there is a unitary operator $V \in \mathcal{L}(\mathcal{H})$ such that $T=V^{*} S V$ which implies that $T^{*}=V^{*} S^{*} V$. Noting that $T^{n}=V^{*} S^{n} V$ and $\left(T^{*} T\right)^{k}=V^{*}\left(S^{*} S\right)^{k} V$. Inserting $I=V V^{*}$ suitably, we deduce from that

$$
V^{*} S^{n}\left(S^{*} S\right)^{k} V=T^{n}\left(T^{*} T\right)^{k}=\left(T^{*} T\right)^{k} T^{n}=V^{*}\left(S^{*} S\right)^{k} S^{n} V,
$$


and follows for $S$.

Proposition 3.6. If $T$ is both of class $[(n, k) Q N]$ and $[(n+1, k) Q N]$ such that $T$ is injective, then $T$ is $k$-quasi-normal.

Proof. Since $T$ is of class $[(n, k) Q N] \cap[(n+1, k) Q N]$, we have by (, which reads $T^{n}\left(T\left(T^{*} T\right)^{k}-\left(T^{*} T\right)^{k} T\right)=0$. If $T$ is injective, then so is $T^{n}$ and we have $T\left(T^{*} T\right)^{k}-\left(T^{*} T\right)^{k} T=0$, whence $T$ is $k$-quasi-normal.

Proposition 3.7. Let $T \in \mathcal{L}(\mathcal{H})$ such that $T$ is of class $[(2, k) Q N] \cap[(3$, $k) Q N]$, then $T$ is of class $[(n, k) Q N]$ for all positive integer $n \geq 4$ and $k=$ $1,2, \ldots$.

Proof. We proof the assertion by using the mathematical induction. For $n=4$ it is a consequence of Remark 3.2.

We prove this for $n=5$. Since $T \in[(2, k) Q N]$,

$$
T^{2}\left(T^{*} T\right)^{k}=\left(T^{*} T\right)^{k} T^{2},
$$

multiplying (3.4) to the left by $T^{3}$ we get

$$
T^{5}\left(T^{*} T\right)^{k}=T^{3}\left(T^{*} T\right)^{k} T^{2} .
$$

Thus implies

$$
T^{5}\left(T^{*} T\right)^{k}=\left(T^{*} T\right)^{k} T^{5} .
$$

Now assume that the result is true for $n \geq 5$ i.e

$$
T^{n}\left(T^{*} T\right)^{k}=\left(T^{*} T\right)^{k} T^{n},
$$

then

$$
\begin{aligned}
T^{n+1}\left(T^{*} T\right)^{k} & =T\left(T^{*} T\right)^{k} T^{n} \\
& =T\left(T^{*} T\right)^{k} T^{2} T^{n-2} \\
& =T^{3}\left(T^{*} T\right)^{k} T^{n-2} \\
& =\left(T^{*} T\right)^{k} T^{n+1} .
\end{aligned}
$$

Thus $T$ is of class $[(n+1, k) Q N]$.

The proof of the following proposition is very similar to whose of Proposition 3.7 ,we omit it.

Proposition 3.8. Let $T \in \mathcal{L}(\mathcal{H})$ such that $T$ is of class $[(n, 2) Q N] \bigcap[(n, 3) Q N]$, then $T$ is of class $[(n, k) Q N]$ for all positive integer $n \geq 1$ and $k=2,3, \ldots$. 
Combining Proposition 3.7 and proposition 3.8, we obtain the following theorem

Theorem 3.6. Let $T \in \mathcal{L}(\mathcal{H})$ such that $T$ is of class $[(2,2) Q N] \cap[(3,3) Q N]$, then $T$ is of class $[(n, k) Q N]$ for all positive integer $n \geq 4$ and $k=1,2, \ldots$.

\section{SVEP and Bishop's Property $(\beta)$ for $n$-Power Quasi-Normal Operators}

The invariant subspace problem is the long standing simple question:"Does every bounded linear operator $T \in \mathcal{L}(\mathcal{H})$ have a non-trivial invariant subspace?"

$$
\text { ( i.e., } \exists \text { a closed subspace } \mathcal{M}:\{0\} \neq \mathcal{M} \neq \mathcal{H} \text { such that } T \mathcal{M} \subset \mathcal{M} \text { ? ). }
$$

This problem is open for more than half a century, and mathematicians have been trying to attack this problem producing many significant contributions with a huge variety of techniques, making this challenging problem a target. However the solution seems to be nowhere in sight. In this section, we discuss the greatest achievements in solving this problem for special classes of linear operators. Especially, we obtained that some operators in $[n Q N]$ are subscalar of order $m$ and those operators satisfy Bishop's property $(\beta)$. Since the results are closely related to the nontrivial invariant subspace problem in operator theory. We give our main results and proofs. For this purpose, we need some auxiliary results.

Proposition 4.1. ([25], Proposition 2.1) For every bounded disk $\mathcal{D}$ in $\mathbb{C}$, there is a constant $C_{\mathcal{D}}$ such that for an arbitrary operator $T \in \mathcal{L}(\mathcal{H})$ and $f \in W_{2}^{m}(\mathcal{D}, \mathcal{H})$ we have

$$
\left\|(I-P) \bar{\partial}^{j} f\right\|_{2, D} \leq C_{\mathcal{D}}\left(\left\|(T-\lambda)^{*} \bar{\partial}^{j+1} f\right\|_{2, \mathcal{D}}+\left\|(T-\lambda)^{*} \bar{\partial}^{j+2} f\right\|_{2, \mathcal{D}}\right), j=0,1, \ldots, m-2,
$$

where $P$ is the orthogonal projection of $L^{2}(\mathcal{D}, \mathcal{H})$ onto $A^{2}(\mathcal{D}, \mathcal{H})$.

Next Theorem is the important result for the proof of our main theorem.

Theorem 4.1. Let $\mathcal{D}$ be an arbitrary bounded disk in $\mathbb{C}$. If $T$ is of class $[n Q N]$ such that $\sigma(T) \cap \sigma\left(\omega_{k}^{-1} T\right)=\emptyset$, for $k=1,2, \ldots, n-1$ then the operator

$$
\lambda-T: W_{2}^{m}(\mathcal{D}, \mathcal{H}) \longrightarrow W_{2}^{m}(\mathcal{D}, \mathcal{H})
$$

is one-to-one. 
Proof. Let $g \in W_{2}^{m}(\mathcal{D}, \mathcal{H})$ such that $(\lambda-T) g=0$ i.e.,

$$
\|(\lambda-T) g\|_{W_{2}^{m}(\mathcal{D}, \mathcal{H})}=0 .
$$

Then for $j=0,1, \ldots, m$ we have

$$
\left\|(\lambda-T) \overline{\partial^{j}} g\right\|_{2, \mathcal{D}}=0
$$

Hence for $j=0,1, \ldots, m$ we get

$$
\left\|\left(\lambda^{n}-T^{n}\right) \overline{\partial^{j}} g\right\|_{2 ; \mathcal{D}}=0 .
$$

Since $\sigma(T) \cap\left(\sigma\left(\omega_{k}^{-1} T\right)\right)$ is empty for $k=1,2, \ldots, n-1$, then $T^{n}$ is normal [ [22], Theorem 2.2]. Hence,

$$
\left\|\left(\bar{\lambda}^{n}-T^{* n}\right) \overline{\partial^{j}} g\right\|_{2, \mathcal{D}}=0
$$

Now we claim that

$$
\left\|\left(\bar{\lambda} I-T^{*}\right) \overline{\partial^{j}} g\right\|_{2, \mathcal{D}}=0 .
$$

Indeed, Since $\lambda-T$ is invertible for $\lambda \in \mathcal{D} \backslash \sigma(T)$, the Eq.(4.2) implies that

$$
\left\|\overline{\partial^{j}} g\right\|_{2, \mathcal{D} \backslash \sigma(T)}=0 .
$$

Therefore

$$
\left\|\left(\bar{\lambda}-T^{*}\right) \overline{\partial^{j}} g\right\|_{2, \mathcal{D} \backslash \sigma(T)}=0 .
$$

Since $\sigma(T) \cap\left(\sigma\left(\omega_{k}^{-1} T\right)\right)=\emptyset$ and $\sigma\left(T^{*}\right)=\sigma(T)^{*}, \bar{\lambda}-\overline{\omega_{k}} T^{*}(1 \leq k \leq n-1)$ are invertible for $\lambda \in \sigma(T)$, therefore, from Eq. (4.3) we have

$$
\left\|\left(\bar{\lambda}-T^{*}\right) \bar{\partial}^{j} g\right\|_{2, \sigma(T)}=0
$$

It is clear form Eqs. (4.5), and (4.6) that

$$
\left\|\left(\bar{\lambda}-T^{*}\right) \overline{\partial^{j}} g\right\|_{2, D}=0, \text { for } j=0,1, \ldots, m .
$$

Thus Proposition 4.1 and Eq.(4.6) imply

$$
\left\|(I-P) \bar{\partial}^{j} g\right\|_{2, D}=0, j=0,1, \ldots, m-2
$$

where $P$ denotes the orthogonal projection of $L^{2}(D ; H)$ onto $A^{2}(D ; H)$.

Hence $\left(\lambda^{n}-T^{n}\right) P g=\left(\lambda^{n}-T^{n}\right) g=0$. Since $T^{n}$ has SVEP $g=0$.

Thus, $\lambda-T$ is one- to-one. 
Corollary 4.1. If $T_{1}$ and $T_{2}$ are of class $[n Q N]$ such that $\sigma\left(T_{i}\right) \cap \sigma\left(\omega_{k}^{-1} T_{i}\right)=$ $\emptyset$, for $k=1,2, \ldots, n-1, i=1,2$ and $T_{2} T_{1}=0$. Then

$$
\lambda-T_{1}-T_{2}: W_{2}^{m}(\mathcal{D}, \mathcal{H}) \longrightarrow W_{2}^{m}(\mathcal{D}, \mathcal{H})
$$

is one to one.

Proof. If $f \in W_{2}^{m}(\mathcal{D}, \mathcal{H})$ is such $\left(\lambda-T_{1}-T_{2}\right) f=0$. Since $T_{2} T_{1}=0$, we get $\left(\lambda I-T_{2}\right) T_{2} f=0$. Since $\lambda-T_{2}$ is one-to-one, $T_{2} f=0$. Hence, $\left(\lambda-T_{1}\right) f_{1}=0$. Since $\lambda-T_{1}$ is one to one, $f=0$.

The following corollary shows that the nilpotent perturbation of operators in $[n Q N]$ satisfying SVEP satisfy SVEP.

Corollary 4.2. If an operator $T \in \mathcal{L}(H)$ is a nilpotent perturbation of a $n$-power quasi-normal operator $S$, i.e., $T=S+N$ where $S$ is n-power quasi-normal, $S$ and $N$ commute, $N^{m}=0$ and $\sigma(S) \cap \sigma\left(\omega_{k}^{-1} S\right)=\emptyset$ for $k=$ $1,2, \ldots, n-1$. Then $\lambda-T$ is one-to-one.

Proof. Let $g \in W_{2}^{m}(\mathcal{D}, \mathcal{H})$ is such that $(\lambda-T) g=0$, then

$$
(\lambda-S) g=N g \text {. }
$$

Hence $(\lambda-S) N^{j-1} g=N^{j} g \quad$ for $j=1,2, \ldots, m$. We prove that $N^{j} g=0$ for $j=0,1, \ldots, m-1$ by indication. Since $N^{m}=0,(\lambda-S) N^{m-1} g=N^{m} g=0$. As $\lambda-S$ is one-to-one by Theorem 4.1 we have $N^{m-1} g=0$. Assume it is true when $j=k$, i.e.. $N^{k} g=0$. From Eq.(4.7), we get

$$
(\lambda-S) N^{k-1} g=N^{k} g=0 .
$$

Since $\lambda-S$ is one-to-one from Theorem $4.1 N^{k-1} g=0$. By indication we have $g=0$. Hence $\lambda-T$ is one-to-one.

Corollary 4.3. If $T$ is of class $[n Q N]$ with the property $\left.\sigma(T) \cap \sigma\left(\omega_{k}^{-1} T\right)\right)=$ $\emptyset$ for $k=1,2, \ldots, n-1$, and if $S$ is a left invertible operator with the left inverse is $R$ then the operator $\lambda-S T R: W_{2}^{m}(\mathcal{D}, \mathcal{H}) \longrightarrow W_{2}^{m}(\mathcal{D}, \mathcal{H})$, is one-to-one.

Proof. If $g \in W_{2}^{m}(\mathcal{D}, \mathcal{H})$ is such that $(\lambda-S T R) g=0$, then

$$
(\lambda-T) R g=0 .
$$

Hence for $j=0,1, \ldots, m$ we have $(\lambda-T) R \bar{\partial}^{j} g=0$. From Theorem 4.1, we get $R \bar{\partial}^{j} g=0$ for $j=0,1, \ldots, m$.

Thus, $S T R \bar{\partial}^{j} g=0$ for $j=0,1, \ldots, m$. It follows that $\lambda \bar{\partial}^{j} g=0$ for $j=$ $0,1, \ldots, m$. By application of $[26$, Proposition 2.1] with $T=(0)$, we have

$$
\|(I-P) g\|_{2, D}=0,
$$


where $P$ denotes the orthogonal projection of $L^{2}(\mathcal{D}, \mathcal{H})$ onto the Bergman space $A^{2}(\mathcal{D}, \mathcal{H})$. Hence $\lambda g=\lambda P g=0$. From [7, Corollary 10.7], there exists a constant $c>0$ such that

$$
c\|P g\|_{2, \mathcal{D}} \leq\|\lambda g\|_{2, \mathcal{D}} .
$$

So $g=P g=0$. Thus, $\lambda-S T R$ is one-to-one.

Corollary 4.4. If $T$ is of class $[n Q N]$ such that $\sigma(T) \cap \sigma\left(\omega_{k}^{-1} T\right)=\emptyset$, for $k=1,2, \ldots, n-1$ and if $S$ is a right invertible operator with the right inverse is $R$ then the operator $\lambda-R T S: W_{2}^{m}(\mathcal{D}, \mathcal{H}) \longrightarrow W_{2}^{m}(\mathcal{D}, \mathcal{H})$, is one-to-one.

An arbitrary operator $T$ in $\mathcal{L}(\mathcal{H})$ has a unique polar decomposition $T=$ $U|T|$, where $|T|=\left(T^{*} T\right)^{\frac{1}{2}}$ and $U$ is a partial isometry with initial space the closure of the range of $|T|$ and final space the closure of the range of $T$. Associated with $T$ there is a related operator $\widetilde{T}=|T|^{\frac{1}{2}} U|T|^{\frac{1}{2}}$, sometimes called the Aluthge transform of $T$ (see [11] and the reference their in).

Proposition 4.2. If $T$ is of class $[n Q N]$ such that $\sigma(T) \cap \sigma\left(\omega_{k}^{-1} T\right)=\emptyset$, for $k=1,2, \ldots, n-1$. Then

$$
\lambda-\widetilde{T}: W_{2}^{m}(\mathcal{D}, \mathcal{H}) \longrightarrow W_{2}^{m}(\mathcal{D}, \mathcal{H}),
$$

is one-to-one.

Proof. Let $f \in W_{2}^{m}(\mathcal{D}, \mathcal{H})$ be such that $(\lambda-\widetilde{T}) f=0$, then

$$
(\lambda-T) U|T|^{\frac{1}{2}} f=0 .
$$

Since $\lambda-T$ is one-to one by Theorem 4.1, $\left.U\right|^{\frac{1}{2}} f=0$. Hence $\widetilde{T} f=0$. That means $\lambda f=0$. This implies that $\overline{\partial^{j}} f=0$ for $j=0,2, \ldots, m$.

Now apply Proposition 4.1 with $T=0$ we deduce that

$$
\|(I-P) f) \|_{2 . \mathcal{D}} \leq D_{\mathcal{D}}\left(\left\|-\lambda \overline{\partial^{j}} f\right\|_{2 . \mathcal{D}}+\left\|-\lambda \overline{\partial^{j+1}} f\right\|_{2 . \mathcal{D}}\right), j=0,1, \ldots, m-2 .
$$

Using the same argument as in the proof of corollary 4.3, we conclude that $f=0$ and the proof is complete.

Proposition 4.3. Let $T \in \mathcal{L}(\mathcal{H})$ be such that $\sigma(T) \cap \sigma\left(\omega_{k}^{-1} T\right)=\emptyset$, for $k=1,2, \ldots, n-1$. If the Aluthge transform $\widetilde{T}$ of $T$ is of class $[n Q N]$, then

$$
\lambda-T: W_{2}^{m}(\mathcal{D}, \mathcal{H}) \longrightarrow W_{2}^{m}(\mathcal{D}, \mathcal{H})
$$

is one-to -one. 
Proof. Since $\sigma(T)$ and $\sigma(\widetilde{T})$ are identical: $\sigma(T)=\sigma(\widetilde{T})$, we have

$$
\sigma(\widetilde{T}) \cap \sigma\left(\omega_{k}^{-1} \widehat{T}\right)=\emptyset, \text { for } k=1,2, \ldots, n-1 .
$$

The result follows from Proposition 4.2 and the fact that the spectrum of $T$ and $\widetilde{T}$ are identical: $\sigma(T)=\sigma(\widetilde{T})$.

In the following theorem we generalized Theorem 3.27 of [23].

Theorem 4.2. If $T$ is of class $[n Q N]$ with the property that $\sigma(T) \cap$ $\sigma\left(\omega_{k}^{-1} T\right)=\emptyset$ for $k=1,2, \ldots, n-1$ and let $\mathcal{D}$ be a bounded disk which contained $\sigma(T)$. Then the operator $V: \mathcal{H} \longrightarrow \mathcal{H}(\mathcal{D})$, defined by

$$
\left.V g=1 \otimes g+\overline{(\lambda-T) W_{2}^{m}(\mathcal{D}, \mathcal{H})}=\widehat{[1 \otimes g}\right]
$$

is one to one and has closed range, where $H(\mathcal{D})$ is as in $(2.3)$.

Proof. First, we shall prove that if $\left\{g_{k}\right\}_{1}^{\infty} \subset \mathcal{H}(\mathcal{D})$ and $\left\{f_{k}\right\}_{1}^{\infty} \subset W_{2}^{m}(\mathcal{D}, \mathcal{H})$ are sequences such that

$$
\lim _{k \longrightarrow \infty}\left\|1 \otimes g_{k}+(\lambda-T) f_{k}\right\|_{W_{2}^{m}}=0
$$

then $\lim _{k \rightarrow \infty} g_{k}=0$.

By the definition of the norm of a Sobolev space, Eq. (4.8) implies that

$$
\lim _{k \longrightarrow \infty}\left\|(\lambda-T) \bar{\partial}^{j} f_{k}\right\|_{2, D}=0 \text { for } j=0,1, \ldots m
$$

From (4.9) we get

$$
\lim _{k \longrightarrow \infty}\left\|\left(\lambda^{n}-T^{n}\right) \bar{\partial}^{j} f_{k}\right\|_{2, \mathcal{D}}=0 \text { for } j=0,1, \ldots m
$$

Since $T^{n}$ is normal,

$$
\lim _{k \longrightarrow \infty}\left\|\left(\bar{\lambda}^{n}-T^{* n}\right) \bar{\partial}^{j} f_{k}\right\|_{2, \mathcal{D}}=0 \text { for } j=0,1, \ldots . m
$$

Since $\lambda-T$ is invertible for $\lambda \in \mathcal{D} \backslash \sigma(T)$, the equation (4.9) implies that

$$
\lim _{k \rightarrow \infty}\left\|\bar{\partial}^{j} f_{k}\right\|_{2, \mathcal{D} \backslash \sigma(T)}=0
$$

Therefore

$$
\lim _{k \rightarrow \infty}\left\|\left(\bar{\lambda}-T^{*}\right) \bar{\partial}^{j} f_{k}\right\|_{2, \mathcal{D} \backslash \sigma(T)}=0 . \quad j=0,1, \ldots, m
$$


Since $\sigma(T) \cap\left(\sigma\left(\omega_{k}^{-1} T\right)\right)=\emptyset$ for $k=1,2, \ldots, n-1$ and $\sigma\left(T^{*}\right)=\sigma(T)^{*}$ it is clear that

$\bar{\lambda}+\overline{\omega_{k}^{-1}} T^{*}(1 \leq k \leq n-1)$ are invertible for $\lambda \in \sigma(T)$. Therefore from the equation (4.11), we have

$$
\left.\lim _{k \longrightarrow \infty} \|\left(\bar{\lambda}-T^{*}\right) \bar{\partial}^{j} f_{k}\right) \|_{2, \sigma(T)}=0 .
$$

Hence, from (4.12) and (4.13) we get

$$
\left.\lim _{k \longrightarrow \infty} \|\left(\bar{\lambda}-T^{*}\right) \bar{\partial}^{j} f_{k}\right) \|_{2, \mathcal{D}}=0 \quad j=0,1, . ., m .
$$

Then by Proposition 4.1, we have

$$
\left.\lim _{k \longrightarrow \infty} \|(I-P) \bar{\partial}^{j} f_{k}\right) \|_{2, \mathcal{D}}=0, j=0,1, \ldots, m-2
$$

By (4.8) and (4.15), we have

$$
\lim _{k \longrightarrow \infty}\left\|1 \otimes g_{k}+(\lambda-T) P f_{k}\right\|_{2, \mathcal{D}}=0 .
$$

Let $\Gamma$ be a curve in $\mathcal{D}$ surrounding $\sigma(T)$. Then for $\lambda \in \Gamma$,

$$
\lim _{k \longrightarrow \infty}\left\|P f_{k}+(\lambda-T)^{-1}\left(1 \otimes g_{k}\right)\right\|=0
$$

uniformly by Eq. (4.16). Hence by Riesz-Dunford functional calculs

$$
\lim _{k \longrightarrow \infty}\left\|\frac{1}{2 \pi i} \int_{\Gamma} P f_{k}(z) d z+g_{k}\right\|=0 .
$$

But by Cauchy's theorem

$$
\int_{\Gamma} P f_{k}(z) d z=0
$$

Hence $\lim _{k \longrightarrow \infty} g_{k}=0$. Let $g \in \mathcal{N}(V)$, i.e. $V(g)=V(1 \otimes g)=0$. Now considering $g$ as a constant sequence we get $g=0$.

To prove the closedness of $\mathcal{R}(V)$, let $\left\{y_{k}\right\}_{1}^{\infty} \subset R(V)$ be a sequence, with $\lim _{k \rightarrow \infty} y_{k}=y_{0}$. Then there is a sequence $\left\{x_{k}\right\}_{1}^{\infty} \subset H$ such that $y_{k}=V\left(x_{k}\right)=$ $V\left(1 \otimes x_{k}\right)$. The sequence $\left\{y_{k}\right\}$ is fundamental, i.e, $\lim _{k \rightarrow \infty}\left\|y_{k}-y_{k+p}\right\|_{W_{2}^{m}}=0$. for every $p \in \mathbb{N}$. Then there is a sequence $\left\{g_{k}\right\} \subset W_{2}^{m}(\mathcal{D}, \mathcal{H})$, such that

$$
\lim _{k \longrightarrow \infty}\left\|1 \otimes\left(x_{k}-x_{k+p}\right)+(\lambda-T)\left(f_{k}-f_{k+p}\right)\right\|=0 \forall p \in \mathbb{N} .
$$


By the proven above it follows $\lim _{k \longrightarrow \infty}\left\|x_{k}-x_{k+p}\right\|=0 ; \quad \forall p \in \mathbb{N}$. So, the sequence $\left\{x_{k}\right\}$ is fundamental. Let $x_{0}=\lim _{k \rightarrow \infty} x_{k}$. Then $V\left(1 \otimes x_{k}\right) \longrightarrow V\left(1 \otimes x_{0}\right)$ when $k \longrightarrow \infty$, and hence $y_{0}=V\left(1 \otimes x_{0}\right)$. Thus, the map $V$ is one-to-one and has closed range.

Corollary 4.5. If $T$ is of class $[n Q N]$ such that $\left.\sigma(T) \cap \sigma\left(\omega_{k}^{-1} T\right)\right)=\emptyset$ for $k=1,2, \ldots, n-1$, then $T$ is subscalar of order $m$.

Proof. Consider an arbitrary bounded open disk $\mathcal{D}$ in $\mathbb{C}$ that contains $\sigma(T)$ and the quotient space given in (2.3).

Let $M_{\lambda}$ be the multiplication operator by $\lambda$ on $W_{2}^{m}(\mathcal{D}, \mathcal{H})$. Then $M_{\lambda}$ is a scalar operator of order $m$ and its spectral distribution is

$$
\Phi_{M}: C_{0}^{m}(\mathbb{C}) \longrightarrow \mathcal{L}\left(W_{2}^{m}(D ; H)\right) ; \Phi_{M}(f)=M_{f},
$$

where $M_{f}$ is the multiplication operator by $f \in C_{0}^{m}(\mathbb{C})$. Let $S \equiv \widehat{M}$. Since $(\lambda-T) W_{2}^{m}(\mathcal{D} ; \mathcal{H})$ is invariant under every operator $M_{f}$, we infer that $S$ is a scalar operator of order $m$ with spectral distribution $\widehat{\Phi}$.

Let $V$ be the operator

$$
V g=1 \otimes g+\overline{(\lambda-T) W_{2}^{m}(\mathcal{D}, \mathcal{H})}
$$

from $\mathcal{H}$ into $\mathcal{H}(\mathcal{D})$. Then we have the following commutative diagram

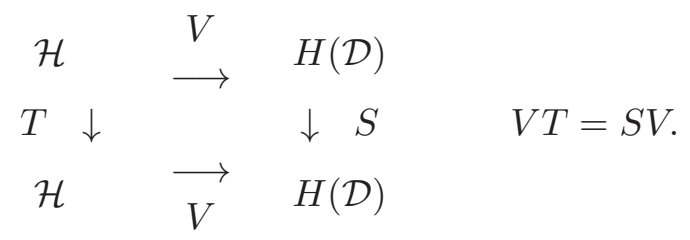

By the previous Theorem the operator $V$ is a topological isomorphism of $H$ into $\mathcal{R}(V)$. The relation $V T=S V$ shows that $R(V)$ is $S$-invariant. Hence $S$ is an extension of the operator $V$, so this operator is subscalar. Since $V$ is invertible on $\mathcal{R}(V)$, then the operator $T$ is subscalar of order $m$. The theorem is proved.

Corollary 4.6. If $T$ is of class $[n Q N]$ such that $\left.\sigma(T) \cap \sigma\left(\omega_{k}^{-1} T\right)\right)=\emptyset, k=$ $1,2, \ldots, n-1$, then $T$ has Bishop's property $(\beta)$.

Proof. It follows from Corollary 4.5 and [15, Lemma 2.1].

Corollary 4.7. Let $T \in \mathcal{L}\left(\oplus_{i=1}^{k} \mathcal{H}\right)$ be the following $k \times k$ triangular operator matrix 


$$
\left(\begin{array}{cccccc}
T_{11} & T_{12} & T_{13} & \ldots & \ldots & T_{1 k} \\
0 & T_{22} & T_{23} & \ldots & \ldots & T_{2 k} \\
0 & 0 & T_{33} & \ldots & \ldots & T_{3 k} \\
0 & 0 & 0 & \ldots & \ldots & \ldots \\
0 & \ldots & \ldots & \ldots & \ldots & T_{k k}
\end{array}\right)
$$

Assume that $T_{i i}$ is of class $[n Q N]$ and satisfies $\left.\sigma\left(T_{i i}\right) \cap \sigma\left(\omega_{p}^{-1} T_{i i}\right)\right)=\emptyset$ for $1 \leq p \leq n-1$ and $i=1,2, \ldots, k$. Then

$$
\lambda-T: W_{2}^{m}\left(\mathcal{D}, \oplus_{i=1}^{k} \mathcal{H}\right) \longrightarrow W_{2}^{m}\left(\mathcal{D}, \oplus_{i=1}^{k} \mathcal{H}\right)
$$

is one-to-one.

Proof. Let $f=\oplus_{i=1}^{k} f_{i} \in \oplus_{i=1}^{k} \mathcal{H}$ is such that $(\lambda-T) f=0$, then we have

$$
\left(\begin{array}{c}
\left(\lambda-T_{11}\right) f_{1}-T_{12} f_{2}-T_{13} f_{3} \cdots-T_{1 k} f_{k} \\
\left(\lambda-T_{22}\right) f_{2}-T_{23} f_{3}-\cdots-T_{2 k} f_{k} \\
\left(\lambda-T_{33}\right) f_{3}-\cdots-T_{3 k} f_{k} \\
\cdots \\
\cdots \\
\cdots \\
\left(\lambda-T_{k-1 k-1}\right) f_{k-1}-T_{k k} f_{k} \\
\left(\lambda-T_{k k}\right) f_{k}
\end{array}\right)=0 .
$$

Hence $\left(\lambda-T_{k k}\right) f_{k}=0$. Since $\lambda-T_{k k}$ is one-to-one from Theorem 3.8, $f_{k}=0$. Then we obtain the following equation $\left(\lambda-T_{k-1 k-1}\right) f_{k-1}=0$. Since $\lambda-T_{k-1}$ is one to-one from Theorem 4.1, $f_{k-1}=0$. By repeating this process we prove that $f=0$. Hence the result.

Proposition 4.4. Let $T \in \mathcal{L}\left(\oplus_{i=1}^{k} \mathcal{H}\right)$ be the following $k \times k$ triangular operator matrix

$$
T=\left(\begin{array}{cccccc}
T_{11} & T_{12} & T_{13} & \ldots & \ldots & T_{1 k} \\
0 & T_{22} & T_{23} & \ldots & \ldots & T_{2 k} \\
0 & 0 & T_{33} & \ldots & \ldots & T_{3 k} \\
0 & 0 & 0 & \ldots & \ldots & \ldots \\
0 & \ldots & \ldots & \ldots & \ldots & T_{k k}
\end{array}\right)
$$

Assume that $T_{i i}$ is of class $[n Q N]$ and satisfies $\sigma\left(T_{i i}\right) \cap\left(\sigma\left(\omega_{q}^{-1} T_{i i}\right)\right)=\emptyset$ for $1 \leq q \leq n-1, i=1,2, \ldots, k-1$ and $T_{k k}$ is nilpotent. Then $T$ has Bishop's property $(\beta)$ and Dunford's property $(C)$. 
Proof. Let $f_{p}=\oplus_{i=1}^{k} f_{p}^{i}: \mathcal{D} \longrightarrow \oplus_{i=1}^{k} \mathcal{H}$ is a sequence of analytic functions such that $(\lambda-T) f_{p}(\lambda) \longrightarrow 0$ uniformly on every compact subset $K$ of an open set $\mathcal{D}$ of $\mathbb{C}$, then we have

$$
\left\{\begin{array}{c}
\left.\left(\lambda-T_{11}\right) f_{p}^{1}(\lambda)+T_{12}\right) f_{p}^{2}(\lambda)+T_{13} f_{p}^{3}(\lambda) \cdots+T_{1 k} f_{p}^{k}(\lambda) \longrightarrow 0 \\
\left(\lambda-T_{22}\right) f_{p}^{2}(\lambda)+T_{23} f_{p}^{3}(\lambda)+\cdots+T_{2 k} f_{p}^{k} \longrightarrow 0 \\
\left(\lambda-T_{33}\right) f_{p}^{3}(\lambda)+\cdots+T_{3 k} f_{p}^{k}(\lambda) \longrightarrow 0 \\
\cdots \\
\cdots \\
\cdots \\
\left(\lambda-T_{k-1 k-1}\right) f_{p}^{k-1}(\lambda)+T_{k-1 k} f_{p}^{k}(\lambda) \longrightarrow 0 \\
\left(\lambda-T_{k k}\right) f_{p}^{k}(\lambda) \longrightarrow 0
\end{array}\right.
$$

Since $T_{k k}^{m}=0, \lambda T_{k k}^{m-1} f_{p}^{k}(\lambda) \longrightarrow 0$ and hence $T_{k k}^{m-1} f_{p}^{k}(\lambda) \longrightarrow 0$ if $\lambda \neq 0$. Since $\left(\lambda-T_{k k}\right) f_{p}^{k}(\lambda) \longrightarrow 0$ from (4.17) $\lambda T_{k k}^{m-2} f_{p}^{k}(\lambda) \longrightarrow 0$. By the same reason, $T_{k k}^{m-3} f_{p}^{k}(\lambda) \longrightarrow 0$. By repeating this procedure, we finally achieve

$$
f_{p}^{k}(\lambda) \longrightarrow 0
$$

uniformly on $K$. Then we obtain the following equation $\left(\lambda-T_{k-1 k-1}\right) f_{p}^{k-1} \longrightarrow 0$ uniformly on every compact $K$. Since $T_{k-1 k-1}$ has Bishop's property $(\beta)$ from Corollary $4.6, f_{p}^{k-1}(\lambda) \longrightarrow 0$ uniformly on $K$. By repeating this process we prove that $f_{p}^{1}(\lambda) \longrightarrow 0$ uniformly on $K$.

Hence $\left\{f_{p}=f_{p}^{1} \oplus f_{p}^{2} \oplus \ldots \oplus f_{p}^{k}\right\}$ converge uniformly to 0 on any compact subset $K$ of $\mathcal{D}$, and so $T$ has the Bishop's property $(\beta)$.

\section{Bishop's Property $(\beta)$ for the Berberian Extension}

There exists an Hilbert space $H^{\circ}: H \subset H^{\circ}$, and an isometric ${ }^{*}$-homomorphism preserving order, i.e, for all $T, S \in \mathcal{L}(H)$ and $\lambda, \mu \in \mathbb{C}$, we have

Proposition 5.1. ( [4] Berberian extension) Let $H$ be a complex Hilbert space. Then there exists a Hilbert space $H^{\circ} \supset H$ and a map

$$
\Phi: \mathcal{L}(H) \rightarrow \mathcal{L}\left(H^{\circ}\right): T \longmapsto T^{\circ}
$$

satisfying: $\Phi$ is an ${ }^{*}$-isometric isomorphism preserving the order such that 
1. $\Phi\left(T^{*}\right)=\Phi(T)^{*}$.

2. $\Phi(\lambda T+\mu S)=\lambda \Phi(T)+\mu \Phi(S)$.

3. $\Phi\left(I_{H}\right)=I_{H^{\circ}}$.

4. $\Phi(T S)=\Phi(T) \Phi(S)$.

5. $\|\Phi(T)\|=\|T\|$.

6. $\Phi(T) \leq \Phi(S)$ if $T \leq S$.

7. $\sigma(\Phi(T))=\sigma(T), \quad \sigma_{a}(T)=\sigma_{a}(\Phi(T))=\sigma_{p}(\Phi(T))$.

8. If $T$ is a positive operator, then $\Phi\left(T^{\alpha}\right)=|\Phi(T)|^{\alpha}$ for all $\alpha>0$.

Lemma 5.1. ([22]) If $T$ is of class $[n Q N]$ then $\Phi(T)$ is of class $[n Q N]$.

Theorem 5.1. If $T$ is of class $[n Q N]$ such that $\sigma(T) \cap \sigma\left(\omega_{k}^{-1} T\right)=\emptyset$ for $k=1,2, \ldots, n-1$. Then $T^{\circ}$ has Bishop's property $(\beta)$.

Proof. Form Lemma 5.1 and Theorem 5.1 we have $T^{\circ}$ is of class $[n Q N]$ and $\sigma\left(T^{\circ}\right) \cap \sigma\left(\omega_{k}^{-1} T^{\circ}\right)=\sigma\left(T^{\circ}\right) \cap \sigma\left(\left(\omega_{k}^{-1} T\right)^{\circ}\right)=\sigma(T) \cap \sigma\left(\omega_{k}^{-1} T\right)=\emptyset ; k=1,2, \ldots, n-1$. Hence Corollary 4.6 ensures that $T^{\circ}$ has Bishop's property $(\beta)$.

\section{Bishop's Property $(\beta)$ for $m$-Partial Isometries}

Contrary to the case of quasi-normal operators, in general, the kernel of an $\mathrm{m}$-partial isometry is not reducing. The following proposition gives a more detailed description of Several spectral properties of some m- partial isometries, concerning the approximate spectrum the spectrum and the point spectrum.

Proposition 6.1. ([23], [27])

Let $T \in \mathcal{L}(\mathcal{H})$ be an m-partial isometry such that $\mathcal{N}(T)$ is a reducing subspace for $T$. The following assertions hold.

1. $\lambda \in \sigma_{a p}(T) \backslash\{0\}$ implies $\bar{\lambda} \in \sigma_{a p}\left(T^{*}\right)$, i.e., if $(T-\lambda) x_{n} \longrightarrow 0$ for some sequence of bounded vectors $\left(x_{n}\right) \subset \mathcal{H}$, then $(T-\lambda)^{*} x_{n} \longrightarrow 0$.

2. $\lambda \in \sigma_{p}(T) \backslash\{0\}$ implies $\bar{\lambda} \in \sigma_{p}\left(T^{*}\right)$. 
3. Eigenvectors of $T$ corresponding to distinct eigenvalues are orthogonal, i.e.

$$
\mathcal{N}(T-\lambda) \perp \mathcal{N}(T-\mu) \quad \text { if } \lambda, \mu \in \sigma_{p}(T), \lambda \neq \mu
$$

4. If $\lambda, \mu \in \sigma_{a p}(T)$ and $\left(x_{n}\right),\left(y_{n}\right)$ be sequences of bounded vectors in $\mathcal{H}$ such that $\lambda \neq \mu$

and

$$
\left\|(T-\lambda) x_{n}\right\| \longrightarrow 0,\left\|(T-\mu) y_{n}\right\| \longrightarrow 0 \quad(\text { as } n \longrightarrow \infty)
$$

Then we have

$$
\left\langle x_{n} \mid y_{n}\right\rangle \longrightarrow 0(\text { as } n \longrightarrow \infty) \text {. }
$$

Theorem 6.1. ([23]) If $T \in \mathcal{L}(\mathcal{H})$ be an m-partial isometry such that $\mathcal{N}(T)$ is a reducing subspace for $T$, then $T$ has $S V E P$.

Remark 6.1. It is will known that for $T \in \mathcal{L}(\mathcal{H}), T$ is a partial isometry if and only if $T^{*}$ is so. This equivalence is false for the class of $m$-partial isometry for $m \geq 2$, as shown in the following example

Example 6.1. Let $T=\left(\begin{array}{ccc}0 & 0 & 0 \\ \sqrt{\frac{2}{3}} & 0 & 0 \\ 0 & \frac{1}{\sqrt{2}} & 0\end{array}\right) \in \mathcal{L}\left(\mathbb{C}^{3}\right) . \quad T$ is a 2-partial isometry but $T^{*}$ is not 2-partial isometry.

Theorem 6.2. Let $T$ be an invertible $m$-partial isometry such that $T^{*}$ is a $m$-partial isometry for $m \geq 2$. Then $T$ has Bishop's property $(\beta)$ if and only if $T^{*}$ has Bishop's property $(\beta)$.

Proof.

$T$ is an invertible $m$ - partial isometry

$$
\begin{aligned}
& \Longleftrightarrow \sum_{j=0}^{m}(-1)^{m-j}\left(\begin{array}{c}
m \\
j
\end{array}\right) T^{* m-j} T^{m-j}=0 \\
& \Longleftrightarrow \sum_{j=0}^{m}(-1)^{m-j}\left(\begin{array}{c}
m \\
j
\end{array}\right)\left(T^{-1}\right)^{* j} T^{m-j}=0 \\
& \Longleftrightarrow \sum_{j=0}^{m}(-1)^{m-j}\left(\begin{array}{c}
m \\
j
\end{array}\right) T^{* m-j}\left(T^{-1}\right)^{j}=0 .
\end{aligned}
$$


and

$$
\begin{aligned}
& T^{*} \text { is an invertible } m-\text { partial isometry } \\
\Longleftrightarrow & \sum_{j=0}^{m}(-1)^{m-j}\left(\begin{array}{c}
m \\
j
\end{array}\right) T^{m-j} T^{* m-j}=0 \\
\Longleftrightarrow & \sum_{j=0}^{m}(-1)^{m-j}\left(\begin{array}{c}
m \\
j
\end{array}\right) T^{m-j}\left(\left(T^{*}\right)^{-1}\right)^{j}=0 .
\end{aligned}
$$

Assume that $T^{*}$ has Bishop's property $(\beta)$ and let $f_{n}: U \longrightarrow \mathcal{H}$ be any sequence of analytic functions on $U$ (any open subset) such that $(\lambda-T) f_{n}(\lambda) \longrightarrow 0$ as $n \longrightarrow \infty$ uniformly on all compact subsets of $\mathrm{U}$.

We have

$$
\begin{aligned}
& \sum_{j=0}^{m}\left(\begin{array}{c}
m \\
j
\end{array}\right)\left(\left(T^{*}\right)^{-1}-\lambda\right)^{j}(\lambda-T)^{m-j} \\
= & \sum_{j=0}^{m} \sum_{r=0}^{j} \sum_{q=0}^{m-j}(-1)^{m-r-q}\left(\begin{array}{c}
m \\
j
\end{array}\right)\left(\begin{array}{l}
j \\
r
\end{array}\right)\left(\begin{array}{c}
m-j \\
q
\end{array}\right)\left(\left(T^{*}\right)^{-1}\right)^{r} \lambda^{j+q-r} T^{m-j-q} \\
= & \sum_{j=0}^{m}(-1)^{j}\left(\begin{array}{c}
m \\
j
\end{array}\right)\left(\left(T^{*}\right)^{-1}\right)^{j} T^{m-j}
\end{aligned}
$$

Hence

$$
\begin{aligned}
& \sum_{j=0}^{m}(-1)^{j}\left(\begin{array}{c}
m \\
j
\end{array}\right)\left(\left(T^{*}\right)^{-1}\right)^{j} T^{m-j} f_{n}(\lambda)-\left(\left(T^{*}\right)^{-1}-\lambda\right)^{m} f_{n}(\lambda) \\
= & \sum_{j=0}^{m}\left(\begin{array}{c}
m \\
j
\end{array}\right)\left(\left(T^{*}\right)^{-1}-\lambda\right)^{j}(\lambda-T)^{m-j} f_{n}(\lambda)-\left(\left(T^{*}\right)^{-1}-\lambda\right)^{m} f_{n}(\lambda) \\
= & \sum_{j=0}^{m-1}(-1)^{j}\left(\begin{array}{c}
m \\
j
\end{array}\right)\left(\left(T^{*}\right)^{-1}-\lambda\right)^{j}(\lambda-T)^{m-j} f_{n}(\lambda) \\
= & {\left[\sum_{j=0}^{m-1}\left(\begin{array}{c}
m \\
j
\end{array}\right)\left(\left(T^{*}\right)^{-1}-\lambda\right)^{j}(\lambda-T)^{m-j-1}\right](\lambda-T) f_{n}(\lambda) \longrightarrow 0 }
\end{aligned}
$$

as $n \longrightarrow \infty$ uniformly on all compact subsets of $U$.

We deduce that $\left(\left(T^{*}\right)^{-1}-\lambda\right)^{m} f_{n}(\lambda) \longrightarrow 0$ as $n \longrightarrow \infty$ uniformly on all compact subsets of $U$. 
Since

$$
\left(\left(T^{*}\right)^{-1}-\lambda\right)^{m} f_{n}(\lambda) \longrightarrow 0 \Longleftrightarrow\left(I-\lambda T^{*}\right)^{m} f_{n}(\lambda) \longrightarrow 0
$$

we get $\left(I-\lambda T^{*}\right)^{m} f_{n}(\lambda) \longrightarrow 0$ as $n \longrightarrow \infty$ uniformly on all compact subsets of $U$. Since $T^{*}$ has Bishop's property $(\beta),\left(I-\lambda T^{*}\right)^{m-1} f_{n}(\lambda) \longrightarrow 0$ uniformly on all compact subsets of $U$. By induction we get that $f_{n}(\lambda) \longrightarrow 0$ as $n \longrightarrow \infty$ uniformly on all compact subsets of $U$. Hence, $T$ has Bishop's property $(\beta)$.

\section{Acknowledgements}

The author express his sincere thanks to Professor Salah Mecheri for sending us the papers $[20,21]$.

\section{References}

[1] J. Agler and M. Stankus, $m$-Isometric transformations of Hilbert space I, Integral Equations and Operator Theory,21 (1995), 383-429.

[2] A. Aluthge, On $p$-hyponormal operators for $0<p<1$, Integral Equations Operator Theory 13 (1990) 307-315.

[3] S.C. Arora, P. Arora, On $p$-quasihyponormal operators for $0<p<1$, Yokohama Math. J. 41 (1993) 25-29.

[4] S.K.Berberian, Approximate proper vectors, Proc. Amer. Math. Soc., 13 (1962), 111-114.

[5] S. Brown, Hyponormal operators with thick spectrum have invariant subspaces, Ann. Math. 125 (1987) 93-103.

[6] S.L. Campbell, B.C. Gupta, On k-quasihyponormal operators, Math. Japonoca 23 (1978) 185-189.

[7] J. B. Conway: Subnormal operators. Pitman, London, 1981.

[8] B.P.Duggal, p-hyponormal operator satisfy Bishop's condition $(\beta)$. Integ.eq.oper.theory 40 (2001) 436-440. 
[9] B. P. Duggal, I. H. Jeon, I. H. Kim, On Weyls theorem for quasi-class A operators, J. Korean Math. Soc. 43(4)(2006), 899-909.

[10] T.Furuta, Ito, M., Yamazaki, T.- A subclass of paranormal operators including class of log-hyponormal and several related classes, Sci. Math., 1 (1998), 389-403.

[11] M. Ito, Some classes of operators associated with generalized Aluthge transformation, Sut. J. Math. 35 (1999), 149-165.

[12] I. H. Jeon and I. H. Kim, On operators satisfying $T^{*}\left|T^{2}\right| T \geq T^{*}|T|^{2} T$. Linear Alg. Appl. 418 (2006), 854-862. doi.org/10.1016/j.laa.2006.02.040

[13] S.Jung, E.Ko and M.J.Leo, On class $\mathcal{A}$ operators. Studia Math. 198 (2010), 249-260.

[14] S.Jung, E.Ko and M.J.Leo, Subscalarity of $(p, k)$-quasihyponormal operators. J. Math. Anal. Appl. 380 (2011) 76-86. doi.org/10.1016/j.jmaa.2011.02.063

[15] M.K. Kim and E.Ko, Square roots of hyponormal operators, Glasgow Math. J. 41 (1999) 463-470.

[16] I.H. Kim,On $(p, k)$-quasihyponormal operators. Mathematical inequalities and Applications. Vol. 7,Number 4 (2004),629-638.

[17] E.Ko, k-Quasihyponormal Operators are Subscalar, Integral Equations Operator Theory 28, No.4, 1997, 492-499.

[18] E.Ko and Y.Yang. On Totally *-Paranormal Operators. Czechoslovak Mathematical Journal, Vol. 56 (2006), No. 4, 1265-1280.

[19] K.B. Laursen and M.N. Neumann, Introduction to Local Spectral Theory, Clarendon Press, Oxford,2000.

[20] Mecheri, S. Isolated points of spectrum of $k$-quasi-*-class operators. Stud. Math.. 208, 87-96 (2012).

[21] Mecheri, S. Bishop's property ( $\beta$ ), Hypercyclicity and Hyperinvariant subspaces. Operators and Matrices. Preprint.

[22] Ould Ahmed Mahmoud Sid Ahmed, On the class of $n$-Power Quasi-normal Operators on Hilbert spaces, Bull. Math. Anal. Appl. 3(2011),No 2, 213228. 
[23] Ould Ahmed Mahmoud Sid Ahmed, On Some Normality-Like properties and Bishop's property $(\beta)$ for a class of operators on Hilberet Spaces.International Journal of Mathematics and Mathematical Sciences, Volume 2012. doi:10.1155/2012/975745.

[24] Pietro Aiena, Fredholm and Local Spectral Theory with Applications to Multipliers, Kluwer, 2004.

[25] M. Putinar, Hyponormal operators are subscalar, J. Operator Theory 12 (1984) 385-395.

[26] M.H.M.Rachid, Property $(\omega)$ and quasi-class $(\mathcal{A}, k)$ operators. Revista de unión Mathemática argentina. volume 22, Númmero1, 2011,133-142.

[27] A. Saddi and Ould Ahmed Mahmoud Sid Ahmed m-Partial Isometries on Hilbert Spaces International Journal of functional Analysis, Operators Theory and Applications, 2(2010), No 1, 67-83.

[28] M. Scott, 3-Isometries, Thesis. University of California, San Diego (1987).

[29] D.Senthilkumar,P.M. Naik and R. Santhi,k-Quasi-normal operators. International Journal of Mathematics and Computation.Vol.15,issue No.2, Year 2012.

[30] K. Tanahashi, I.H. Jeon, I. H. Kim and A. Uchiyama, Quasinilpotent part of class A or (p, k)-quasihyponormal operators, Operator Theory: Advances and Applications 187(2008), 199-210.

[31] D.Xia, Spectral Theory of Hyponormal Operators, Birkhäuser Verlag, Basel (1983). 